Article

\title{
Normal Toeplitz Operators on the Fock Spaces
}

\author{
Jongrak Lee $\mathbb{D}$ \\ Department of Mathematics, Jeju National University, Jeju 63243, Korea; jrlee@jejunu.ac.kr
}

Received: 18 September 2020; Accepted: 28 September 2020; Published: 29 September 2020

\begin{abstract}
We characterize normal Toeplitz operator on the Fock spaces $F^{2}(\mathbb{C})$. First, we state basic properties for Toeplitz operator $T_{\varphi}$ on $F^{2}(\mathbb{C})$. Next, we study the normal Toeplitz operator $T_{\varphi}$ on $F^{2}(\mathbb{C})$ in terms of harmonic symbols $\varphi$. Finally, we characterize the normal Toeplitz operators $T_{\varphi}$ with non-harmonic symbols acting on $F^{2}(\mathbb{C})$.
\end{abstract}

Keywords: Toeplitz operator; normal operator; Fock spaces

MSC: Primary 47B35, 47B15. Secondary 30H20

\section{Introduction}

In this paper, we study the normality of Toeplitz operators operating on the Fock space. Our interest is focused on Toeplitz operators with harmonic and non-harmonic symbols.

Many authors in [1-7] studied intensively Normal operators and Toeplitz operators on the Hilbert spaces. It is natural for Toeplitz operators to ask when they are going to be normal. In 1963, Brown and Halmos [8] characterized normal Toeplitz operators on the Hardy space. This contains many basic results of the algebraic properties of Toeplitz operators. It has had significance in operator theory. Thus, we will focus on normal Toeplitz operators with various symbols on the Fock space.

Recently, Kim and Lee [6] gave a characterization for the normality of Toeplitz operators with non-harmonic symbols on the Bergman space. In view of this, we characterize the normal Toeplitz operators with harmonic and non-harmonic symbols acting on the Fock spaces.

Let $\mathcal{H}$ be a separable complex Hilbert space and $\mathcal{L}(\mathcal{H})$ be the algebra of bounded linear operators on $\mathcal{H}$. For any operator $T \in \mathcal{L}(\mathcal{H}), T$ is normal if its self-commutator $\left[T^{*}, T\right]:=T^{*} T-T T^{*}=0$, where $T^{*}$ denotes the adjoint of $T$.

Let $L^{2}(\mathbb{C}, d \mu)$ represents the Hilbert space of all Lebesgue measurable square integrable functions $f$ on the complex plane. For $f \in L^{2}(\mathbb{C}, d \mu)$, the norm of $f$ is denoted by

$$
\|f\|^{2}=\int_{\mathbb{C}}|f(z)|^{2} d \mu(z) .
$$

Here, $d \mu(z)=e^{-|z|^{2}} d A(z)$ and $d A$ is the Lebesgue area measure on $\mathbb{C}$. The Fock spaces $F^{2}(\mathbb{C})$ is the closed subspace of $L^{2}(\mathbb{C}, d \mu)$ comprising all analytic functions in $L^{2}(\mathbb{C}, d \mu)([9]) . F^{2}(\mathbb{C})$ is the Hilbert space with inner product

$$
\langle f, g\rangle=\int_{\mathbb{C}} f(z) \overline{g(z)} d \mu(z)
$$

where $f, g \in F^{2}(\mathbb{C})$. In [9], the author checked that $e_{n}(z)=\frac{z^{n}}{\sqrt{\pi n !}}$ is an orthonormal basis for $F^{2}(\mathbb{C})$ for a nonnegative integer $n$.

For $\varphi \in L^{2}(\mathbb{C})$, the Toeplitz operator with symbol $\varphi$ is the operator $T_{\varphi}$ on $F^{2}(\mathbb{C})$ defined by

$$
T_{\varphi} f:=P(\varphi \cdot f) \quad\left(f \in F^{2}(\mathbb{C})\right) .
$$


Here, $P: L^{2}(\mathbb{C}, d \mu) \rightarrow F^{2}(\mathbb{C})$ represents the orthogonal projection. For any complex numbers $z$, $\omega$, the reproducing kernel in $F^{2}(\mathbb{C})$ is provided by $K_{z}(\omega)=\frac{1}{\pi} e^{\omega \bar{z}}$, and $k_{z}(\omega)=\frac{1}{\sqrt{\pi}} e^{\omega \bar{z}-\frac{|z|^{2}}{2}}$ is the normalized reproducing kernel.

Now, we study the normality of $T_{\varphi}$ on the Fock spaces with various symbols. The following properties are very well-known results of the Toeplitz operators on the Fock space. Let $f, g$ be in $L^{2}(\mathbb{C})$ and $\alpha, \beta \in \mathbb{C}$, then we can easily check that

$$
\begin{aligned}
& \text { (i) } T_{\alpha f+\beta g}=\alpha T_{f}+\beta T_{g} \\
& \text { (ii) } T_{f}^{*}=T_{\bar{f}} \\
& \text { (iii) } T_{\bar{f}} T_{g}=T_{\bar{f} g^{\prime}} \text { if } f \text { or } g \text { is analytic. }
\end{aligned}
$$

This paper is designed as follows. First, we study the basic properties of Toeplitz operators on the Fock spaces and consider the normal Toeplitz operator $T_{\varphi}$ on $F^{2}(\mathbb{C})$ with harmonic symbols $\varphi$. Second, we focus on the normality of Toeplitz operators $T_{\varphi}$ with non-harmonic symbols acting on $F^{2}(\mathbb{C})$ and their applications.

\section{Toeplitz Operators with Harmonic Symbols}

First, we prove the basic results of $T_{\varphi}$ on the Fock spaces. We need several auxiliary lemmas to prove the main theorems. We begin with:

Lemma 1. ([10]) For any nonnegative integers $s, t$,

$$
\left\langle z^{t}, z^{s}\right\rangle=\left\{\begin{array}{ll}
\pi s ! & \text { if } s=t \\
0 & \text { if } s \neq t
\end{array} \text { and } \quad P\left(\bar{z}^{t} z^{s}\right)= \begin{cases}\frac{s !}{(s-t) !} z^{s-t} & \text { if } s \geq t \\
0 & \text { if } s<t\end{cases}\right.
$$

Lemma 2. ([10]) For $m \geq 0$ and $c_{i} \in \mathbb{C}$, we have

(i) $\left\|\bar{z}^{m} \sum_{i=0}^{\infty} c_{i} z^{i}\right\|^{2}=\sum_{i=0}^{\infty} \pi(i+m) !\left|c_{i}\right|^{2}$ and

(ii) $\left\|P\left(\bar{z}^{m} \sum_{i=0}^{\infty} c_{i} z^{i}\right)\right\|^{2}=\sum_{i=m}^{\infty} \frac{\pi(i !)^{2}}{(i-m) !}\left|c_{i}\right|^{2}$.

The following theorem is the characterization of normal Toeplitz operators with harmonic symbols on $F^{2}(\mathbb{C})$.

Theorem 1. Let $\varphi(z)=\overline{g(z)}+f(z)$, where

$$
f(z)=\sum_{n=0}^{N} a_{n} z^{n} \quad \text { and } \quad g(z)=\sum_{n=1}^{m} a_{-n} z^{n}
$$

with $a_{-m} a_{N} \neq 0$. Then, $T_{\varphi}$ is normal on $F^{2}(\mathbb{C})$ if and only if $g(z)=e^{i \theta} f(z)$ for any $\theta \in[0,2 \pi)$.

Proof. Observe that $T_{\varphi}$ is normal if and only if

$$
T_{\bar{f} f}+T_{g} T_{\bar{g}}=T_{f} T_{\bar{f}}+T_{\bar{g} g} .
$$

First, we show that $N=m$. We assume $N>m$. Then, by $z^{m}$ acting on both sides in (1), we have $T_{\bar{f} f} z^{m}=T_{f} T_{\bar{f}} z^{m}$, and hence

$$
P\left(\sum_{n=0}^{N}{\overline{a_{n}}}^{n} \sum_{n=0}^{N} a_{n} z^{n} z^{m}\right)=\sum_{n=0}^{N} a_{n} z^{n} P\left(\sum_{n=0}^{N}{\overline{a_{n}}}^{n} z^{m}\right) .
$$


By direct calculations, we have

$$
P\left(\sum_{n=0}^{N} \overline{a_{n} z^{n}} \sum_{n=0}^{N} a_{n} z^{n} z^{m}\right)=P\left(\sum_{i=0}^{N} \sum_{j=\max \{0, i-m\}}^{N}{\overline{a_{i}}}^{i} a_{j} z^{j+m}\right)
$$

and

$$
\sum_{n=0}^{N} a_{n} z^{n} P\left(\sum_{n=0}^{N}{\overline{a_{n}}}^{n} z^{m}\right)=\sum_{n=0}^{N} a_{n} z^{n} P\left(\sum_{n=0}^{m}{\overline{a_{n}}}^{n} z^{m}\right) .
$$

Looking at the coefficient of $z^{m}$, we deduce that

$$
\sum_{j=0}^{N} \frac{(m+j) !}{m !}\left|a_{j}\right|^{2}=\sum_{j=0}^{m} \frac{m !}{(m-j) !}\left|a_{j}\right|^{2}
$$

Moreover, since

$$
\frac{(m+j) !}{m !} \geq \frac{m !}{(m-j) !}
$$

for $1 \leq j \leq m$ and $\frac{(m+j) !}{m !}>0$ for $m+1 \leq j \leq N$, thus $a_{j}=0$ for all $1 \leq j \leq N$. This is a contradiction to the assumption $a_{N} \neq 0$.

Next, we find the necessary and sufficient condition of normality of $T_{\varphi}$. For any $k \in \mathbb{N}$,

$$
\begin{aligned}
\left(T_{\bar{f} f}-T_{f} T_{\bar{f}}\right) z^{k} & =P\left(\sum_{n=0}^{N} \overline{a_{n} \bar{z}^{n}} \sum_{n=0}^{N} a_{n} z^{n} z^{k}\right)-\sum_{n=0}^{N} a_{n} z^{n} P\left(\sum_{n=0}^{N} \overline{a_{n} z^{n} z^{k}}\right) \\
& =\sum_{i=0}^{\min \{j+k, N\}} \sum_{j=0}^{N} \frac{(k+j) !}{(k+j-i) !} \overline{a_{i}} a_{j} z^{j+k-i}-\sum_{j=0}^{N} a_{j} z^{j} \sum_{i=0}^{k} \frac{k !}{(k-i) !} \bar{a}_{i} z^{k-i}
\end{aligned}
$$

and

$$
\begin{aligned}
\left(T_{\bar{g} g}-T_{g} T_{\bar{g}}\right) z^{k} & =P\left(\sum_{n=1}^{N} \overline{a_{-n} z^{n}} \sum_{n=1}^{N} a_{-n} z^{n} z^{k}\right)-\sum_{n=1}^{N} a_{-n} z^{n} P\left(\sum_{n=1}^{N} \overline{a_{-n} z^{n} z^{k}}\right) \\
& =\sum_{i=1}^{\min \{j+k, N\}} \sum_{j=1}^{N} \frac{(k+j) !}{(k+j-i) !} \overline{a_{-i}} a_{-j} z^{j+k-i}-\sum_{j=1}^{N} a_{-j} z^{j} \sum_{i=1}^{k} \frac{k !}{(k-i) !} \overline{a_{-i}} z^{k-i} .
\end{aligned}
$$

By (1), looking at the coefficients of $z^{k+N-2}$, we have

$$
\begin{aligned}
\overline{a_{0}} & a_{N-2}+\overline{a_{1}} a_{N-1}(N+k-1)+\overline{a_{2}} a_{N}(N+k-1)(N+k) \\
& -\left(\overline{a_{0}} a_{N-2}+\overline{a_{1}} a_{N-1} k+\overline{a_{2}} a_{N} k(k-1)\right) \\
= & \overline{a_{-1}} a_{-(N-1)}(N+k-1)+\overline{a_{-2}} a_{-N}(N+k-1)(N+k) \\
& -\left(\overline{a_{-1}} a_{-(N-1)} k+\overline{a_{-2}} a_{-N} k(k-1)\right)
\end{aligned}
$$

for any $k \in \mathbb{N}$. Therefore,

$$
(N-1)\left(\overline{a_{1}} a_{N-1}-\overline{a_{-1}} a_{-(N-1)}\right)+N(2 k-1)\left(\overline{a_{2}} a_{N}-\overline{a_{-2}} a_{-N}\right)=0
$$

for any $k \in \mathbb{N}$. Since $k$ is arbitrary, we have that $\overline{a_{1}} a_{N-1}=\overline{a_{-1}} a_{-(N-1)}$ and $\overline{a_{2}} a_{N}=\overline{a_{-2}} a_{-N}$. With a similar argument, we have

$$
\overline{a_{i}} a_{j}=\overline{a_{-i}} a_{-j}
$$


for all $i, j$, with $1 \leq i, j \leq N$. Therefore,

$$
a_{-j}=e^{i \theta} a_{j}
$$

for all $1 \leq j \leq N$ and so $g(z)=e^{i \theta} f(z)$.

If $g(z)=e^{i \theta} f(z)$, then

$$
T_{\varphi}^{*} T_{\varphi}=T_{\bar{f}+g} T_{\bar{g}+f}=T_{\bar{f}+e^{i \theta} f} T_{e^{-i \theta} \bar{f}+f}=T_{e^{-i \theta} \bar{f}+f} T_{\bar{f}+e^{i \theta} f}=T_{\varphi} T_{\varphi}^{*} .
$$

Thus, $T_{\varphi}$ is normal on $A^{2}(\mathbb{D})$. This completes the proof.

\section{Toeplitz Operators with Non-Harmonic Symbols}

In this section, we study the normality of $T_{\varphi}$ on $F^{2}(\mathbb{C})$ with non-harmonic symbols. Since symbols of Toeplitz operators cannot be divided into analytic parts and co-analytic parts, the method cannot be applied as in the Theorem 1 . Thus, we have to calculate the self-commutator of $T_{\varphi}$ for non-harmonic symbol $\varphi$. First, we consider the Toeplitz operators with symbol $\varphi$ of the form $\varphi(z)=a_{m, n} z^{m} \bar{z}^{n}$.

Lemma 3. Let $\varphi(z)=a_{m, n} z^{m} \bar{z}^{n}$ with $a_{m, n} \in \mathbb{C}$. Then, $T_{\varphi}$ on $F^{2}(\mathbb{C})$ is normal if and only if $m=n$.

Proof. For $\varphi(z)=a_{m, n} z^{m} \bar{z}^{n}, T_{\varphi}$ is normal if and only if

$$
\left\langle\left(T_{\varphi}^{*} T_{\varphi}-T_{\varphi} T_{\varphi}^{*}\right) \sum_{i=0}^{\infty} c_{i} z^{i}, \sum_{i=0}^{\infty} c_{i} z^{i}\right\rangle=0
$$

for all $c_{i} \in \mathbb{C}$. Using Lemmas 1 and 2, we get that

$$
\begin{aligned}
& \left\|T_{\varphi} \sum_{i=0}^{\infty} c_{i} z^{i}\right\|^{2}-\left\|T_{\varphi}^{*} \sum_{i=0}^{\infty} c_{i} z^{i}\right\|^{2} \\
& =\left\|T_{a_{m, n} z^{m} \bar{z}^{n}} \sum_{i=0}^{\infty} c_{i} z^{i}\right\|^{2}-\left\|T_{\bar{a}_{m, n} \bar{z}^{m} z^{n}} \sum_{i=0}^{\infty} c_{i} z^{i}\right\|^{2} \\
& =\left\|P\left(a_{m, n} z^{m} \bar{z}^{n} \sum_{i=0}^{\infty} c_{i} z^{i}\right)\right\|^{2}-\left\|P\left(\bar{a}_{m, n} \bar{z}^{m} z^{n} \sum_{i=0}^{\infty} c_{i} z^{i}\right)\right\|^{2} \\
& =\left|a_{m, n}\right|^{2} \sum_{i=\max \{n-m, 0\}}^{\infty} \frac{(m+i) !}{(m+i-n) !}\left|c_{i}\right|^{2}-\left|a_{m, n}\right|^{2} \sum_{i=\max \{m-n, 0\}}^{\infty} \frac{(n+i) !}{(n+i-m) !}\left|c_{i}\right|^{2} .
\end{aligned}
$$

Hence, $T_{\varphi}$ is normal if and only if

$$
\sum_{i=\max \{n-m, 0\}}^{\infty} \frac{(m+i) !}{(m+i-n) !}\left|c_{i}\right|^{2}=\sum_{i=\max \{m-n, 0\}}^{\infty} \frac{(n+i) !}{(n+i-m) !}\left|c_{i}\right|^{2}
$$

for all $c_{i} \in \mathbb{C}$. Since $c_{i}$ 's are arbitrary, we can see that $T_{\varphi}$ is normal if and only if $m=n$. This completes the proof.

Now, we consider the normality of Toeplitz operators $T_{\varphi}$ with non-harmonic symbols of two terms. The following consequence gives a general characterization of normal Toeplitz operators with the symbols that are of the form $\varphi(z)=a z^{m} \bar{z}^{n}+b z^{s} \bar{z}^{t}(m \geq n \geq 0, t \geq s \geq 0)$.

Theorem 2. Let $\varphi(z)=a z^{m} \bar{z}^{n}+b z^{s} \bar{z}^{t}$ with $m \geq n \geq 0, t \geq s \geq 0$ and nonzeros $a, b \in \mathbb{C}$. Then, $T_{\varphi}$ is normal if and only if $\varphi(z)$ is either

$$
\varphi(z)=a|z|^{2 m}+b|z|^{2 t} \text { or } \varphi(z)=a\left(z^{m} \bar{z}^{n}+e^{i \theta} z^{n} \bar{z}^{m}\right)
$$

for some $\theta \in[0,2 \pi)$. 
Proof. Let $\varphi(z)=a z^{m} \bar{z}^{n}+b z^{s} \bar{z}^{t}$ with $m \geq n, t \geq s$. By the same arguments as in the proof of Lemma 3, $T_{\varphi}$ is normal if and only if

$$
\begin{aligned}
& \left\|T_{\varphi} \sum_{i=0}^{\infty} c_{i} z^{i}\right\|^{2}-\left\|T_{\varphi}^{*} \sum_{i=0}^{\infty} c_{i} z^{i}\right\|^{2} \\
& =\left\|T_{a z^{m} \bar{z}^{n}} \sum_{i=0}^{\infty} c_{i} z^{i}+T_{b z^{s} \bar{z}^{t}} \sum_{i=0}^{\infty} c_{i} z^{i}\right\|^{2}-\left\|T_{\bar{a} \bar{z}^{m} z^{n}} \sum_{i=0}^{\infty} c_{i} z^{i}+T_{\bar{b} \bar{z}^{s} z^{t}} \sum_{i=0}^{\infty} c_{i} z^{i}\right\|^{2} \\
& =\left\|P\left(a \bar{z}^{n} \sum_{i=0}^{\infty} c_{i} z^{i+m}\right)+P\left(b \bar{z}^{t} \sum_{i=0}^{\infty} c_{i} z^{i+s}\right)\right\|^{2} \\
& -\left\|P\left(\overline{a z}^{m} \sum_{i=0}^{\infty} c_{i} z^{i+n}\right)+P\left(\bar{b} \bar{z}^{s} \sum_{i=0}^{\infty} c_{i} z^{i+t}\right)\right\|^{2} \\
& =|a|^{2} \sum_{i=0}^{\infty} \frac{(m+i) !}{(m+i-n) !}\left|c_{i}\right|^{2}+|b|^{2} \sum_{i=t-s}^{\infty} \frac{(s+i) !}{(s+i-t) !}\left|c_{i}\right|^{2} \\
& -|a|^{2} \sum_{i=m-n}^{\infty} \frac{(n+i) !}{(n+i-m) !}\left|c_{i}\right|^{2}-|b|^{2} \sum_{i=0}^{\infty} \frac{(t+i) !}{(t+i-s) !}\left|c_{i}\right|^{2}=0
\end{aligned}
$$

for any $c_{i} \in \mathbb{C}(i=0,1,2, \ldots)$.

Case (1) If $m=n, t=s$, then the equality (2) holds, and so $T_{\varphi}$ is normal if and only if $\varphi(z)=$ $a|z|^{2 m}+b|z|^{2 t}$.

Case (2) If $m>n, t>s$, put $c_{0}=1$ and $c_{i}=0$ for $i \geq 1$, then

$$
\frac{m !}{(m-n) !}|a|^{2}=\frac{t !}{(t-s) !}|b|^{2}
$$

(i) If $m=n+1, t=s+1$, then we get

$$
\frac{(m+i) !}{(i+1) !}|a|^{2}+\frac{(t+i-1) !}{(i-1) !}|b|^{2}=\frac{(m+i-1) !}{(i-1) !}|a|^{2}+\frac{(t+i) !}{(i+1) !}|b|^{2}
$$

for all $i \geq 1$. From equality (3), we get

$$
\frac{(m+i) !}{(i+1) !} \frac{t !}{m !}+\frac{(t+i-1) !}{(i-1) !}=\frac{(m+i-1) !}{(i-1) !} \frac{t !}{m !}+\frac{(t+i) !}{(i+1) !}
$$

for all $i \geq 1$. Hence, for $i=1$, we have $m=t$ and so $n=s$.

(ii) Let $m>n+1, t=s+1$. Suppose that $T_{\varphi}$ is normal. For a sufficiently large $k$,

$$
\begin{aligned}
T_{\varphi} T_{\bar{\varphi}} z^{k}= & T_{\varphi} P\left(\overline{a z} z^{m} z^{n}+\bar{b} \overline{z^{s}} z^{s+1} z^{k}\right) \\
= & T_{\varphi}\left(\bar{a} \frac{(n+k) !}{(n+k-m) !} z^{n+k-m}+\bar{b} \frac{(s+k+1) !}{(k+1) !} z^{k+1}\right) \\
= & |a|^{2} \frac{(n+k) !^{2}}{k !(n+k-m) !} z^{k}+\bar{a} b \frac{(n+k) !(n+k-m+s) !}{(n+k-m) !(n+k-m-1) !} z^{n+k-m-1} \\
& +a \bar{b} \frac{(s+k+1) !(m+k+1) !}{(k+1) !(m+k-n+1) !} z^{m+k-n+1}+|b|^{2} \frac{(s+k+1) !^{2}}{k !(k+1) !} z^{k},
\end{aligned}
$$


and

$$
\begin{aligned}
T_{\bar{\varphi}} T_{\varphi} z^{k}= & T_{\bar{\varphi}} P\left(a z^{m} \bar{z}^{n} z^{k}+b z^{s} \bar{z}^{s+1} z^{k}\right) \\
= & T_{\bar{\varphi}}\left(a \frac{(m+k) !}{(m+k-n) !} z^{m+k-n}+b \frac{(s+k) !}{(k-1) !} z^{k-1}\right) \\
= & |a|^{2} \frac{(m+k) !^{2}}{k !(m+k-n) !} z^{k}+\bar{a} b \frac{(s+k) !(n+k-1) !}{(k-1) !(n+k-m-1) !} z^{n+k-m-1} \\
& +a \bar{b} \frac{(m+k) !(m+k-n+s+1) !}{(m+k-n) !(m+k-n+1) !} z^{m+k-n+1}+|b|^{2} \frac{(s+k) !^{2}}{(k-1) ! k !} z^{k} .
\end{aligned}
$$

Since $T_{\varphi}$ is normal, we have that

$$
\frac{(s+k+1) !(m+k+1) !}{(k+1) !(m+k-n+1) !}=\frac{(m+k) !(m+k-n+s+1) !}{(m+k-n) !(m+k-n+1) !}
$$

for all sufficiently large $k$. By a direct calculation,

$$
\frac{(s+k+1) !(m+k+1)}{(k+1) !}=\frac{(m+k-n+s+1) !}{(m+k-n) !}
$$

Since $k$ is arbitrary, if

$$
\frac{(s+M+1) !(m+M+1)}{(M+1) !}=\frac{(m+M-n+s+1) !}{(m+m-n) !}
$$

for sufficiently large $M$, then

$$
\begin{aligned}
\frac{(s+M+2) !(m+M+2)}{(M+2) !} & =\frac{(s+M+1) !(m+M+1)}{(M+1) !} \times \frac{(s+M+2)(m+M+2)}{(M+2)(m+M+1)} \\
& =\frac{(m+M-n+s+1) !}{(m+m-n) !} \times \frac{(s+M+2)(m+M+2)}{(M+2)(m+M+1)}
\end{aligned}
$$

and

$$
\frac{(m+M-n+s+2) !}{(m+M-n+1) !}=\frac{(m+M-n+s+1) !}{(m+M-n) !} \times \frac{m+M-n+s+2}{m+M-n+1} .
$$

Therefore,

$$
\frac{(s+M+2)(m+M+2)}{(M+2)(m+M+1)}=\frac{m+M-n+s+2}{m+M-n+1}
$$

and, by a direct calculation, we have

$$
m s-n s-n=0 \text { and } m^{2} s-m n s-2 n-2 n s+m s=0 .
$$

By the first equality, $s=\frac{n}{m-n}$, and so $m n(m-n-1)=0$. Therefore, $m=0$ or $n=0$ or $m=n+1$, a contradiction. Hence, $T_{\varphi}$ is not normal.

(iii) If $m>n+1, t>s+1$, set $c_{1}=1$ and $c_{i}=0$ for $i \neq 1$, then by a similar argument as in (i), $m=t$.

By (i)-(iii), $m=t$ and so $T_{\varphi}$ is normal if and only if

$$
\begin{aligned}
& |a|^{2} \sum_{i=0}^{\infty} \frac{(m+i) !^{2}}{(m+i-n) !}\left|c_{i}\right|^{2}+|b|^{2} \sum_{i=m-s}^{\infty} \frac{(s+i) !}{(s+i-m) !}\left|c_{i}\right|^{2} \\
& \quad-|a|^{2} \sum_{i=m-n}^{\infty} \frac{(n+i) !^{2}}{(n+i-m) !}\left|c_{i}\right|^{2}-|b|^{2} \sum_{i=0}^{\infty} \frac{(m+i) !}{(m+i-s) !}\left|c_{i}\right|^{2}=0
\end{aligned}
$$


for any $c_{i} \in \mathbb{C}(i=0,1,2, \cdots)$. If $n \neq s$, let $n>s$ and from (5) with $c_{1}=1$ and $c_{i}=0$ with $i \neq 1$. If $m-n=1$, then

$$
|a|^{2} \frac{(m+1) !^{2}}{(m-n+1) !}-|a|^{2} \frac{(n+1) !^{2}}{(n-m+1) !}-|b|^{2} \frac{(m+1) !^{2}}{(m-s+1) !}=0,
$$

or, equivalently,

$$
|a|^{2} \frac{(m+1) !^{2}}{2}-|a|^{2} m !^{2}-|b|^{2} \frac{(m+1) !^{2}}{(m-s+1) !}=0 .
$$

By direct calculations with (3), we have

$$
s=m-1-\frac{4}{(m+1)^{2}-2} .
$$

Therefore, $s$ is not nonnegative integer. If $m-n>1$, then

$$
|a|^{2} \frac{(m+1) !^{2}}{(m-n+1) !}-|b|^{2} \frac{(m+1) !^{2}}{(m-s+1) !}=0 .
$$

By (3), we have $n=s$, a contradiction. Therefore, $n=s$ and so

$$
\begin{aligned}
& |a|^{2} \sum_{i=0}^{\infty} \frac{(m+i) !^{2}}{(m+i-n) !}\left|c_{i}\right|^{2}+|b|^{2} \sum_{i=m-n}^{\infty} \frac{(n+i) !^{2}}{(n+i-m) !}\left|c_{i}\right|^{2} \\
& \quad-|a|^{2} \sum_{i=m-n}^{\infty} \frac{(n+i) !^{2}}{(n+i-m) !}\left|c_{i}\right|^{2}-|b|^{2} \sum_{i=0}^{\infty} \frac{(m+i) !^{2}}{(m+i-n)}\left|c_{i}\right|^{2}=0,
\end{aligned}
$$

or, equivalently,

$$
\left(|a|^{2}-|b|^{2}\right)\left(\sum_{i=0}^{\infty} \frac{(m+i) !^{2}}{(m+i-n) !}\left|c_{i}\right|^{2}-\sum_{i=m-n}^{\infty} \frac{(n+i) !^{2}}{(n+i-m) !}\left|c_{i}\right|^{2}\right)=0,
$$

and hence, if $T_{\varphi}$ is normal, then $\varphi(z)=a\left(z^{m} \bar{z}^{n}+e^{i \theta} z^{n} \bar{z}^{m}\right)$ for some $\theta \in[0,2 \pi)$.

If $\varphi$ is the form as $\varphi(z)=a\left(z^{m} \bar{z}^{n}+e^{i \theta} z^{n} \bar{z}^{m}\right)$, i.e., $m=t, n=s$, and $|a|=|b|$, then, by the equalities (2), $T_{\varphi}$ is normal. This completes the proof.

Corollary 1. Let $\varphi(z)=a z^{m}+b \bar{z}^{m}$. Then, $T_{\varphi}$ is normal on $F^{2}(\mathbb{C})$ if and only if $|a|=|b|$.

Next, we will prove the necessary and sufficient conditions for the Toeplitz operator with the sum of the symbols as in Theorem 2 to become a normal Toeplitz operator.

Theorem 3. For $a, b \in \mathbb{C}$, let $\varphi(z)=a \varphi_{1}(z)+b \varphi_{2}(z)$ be of the form

$$
\varphi_{1}(z)=z^{m} \bar{z}^{n}+e^{i \theta_{1}} z^{n} \bar{z}^{m}, \varphi_{2}(z)=z^{s} \bar{z}^{t}+e^{i \theta_{2}} z^{t} \bar{z}^{s},
$$

where $\theta_{1}, \theta_{2} \in[0,2 \pi), m \geq n \geq 0$ and $t \geq s \geq 0$. Then, $T_{\varphi}$ is normal if and only if either

$$
\bar{a} b e^{i \theta_{1}}=a \bar{b} e^{i \theta_{2}}
$$

or

$$
\varphi(z)=a\left(1+e^{i \theta_{1}}\right)|z|^{2 m}+b\left(1+e^{i \theta_{2}}\right)|z|^{2 s} .
$$


Proof. Let $\varphi(z)=a \varphi_{1}(z)+b \varphi_{2}(z)$; then, $T_{\varphi}$ is normal if and only if

$$
T_{a \varphi_{1}} T_{\overline{b \varphi_{2}}}+T_{b \varphi_{2}} T_{\overline{a \varphi_{1}}}=T_{\overline{a \varphi_{1}}} T_{b \varphi_{2}}+T_{\overline{b \varphi_{2}}} T_{a \varphi_{1}}
$$

or, equivalently,

$$
a \bar{b} e^{i \theta_{2}} T_{\varphi_{1}} T_{\varphi_{2}}+\bar{a} b e^{i \theta_{1}} T_{\varphi_{2}} T_{\varphi_{1}}=\bar{a} b e^{i \theta_{1}} T_{\varphi_{1}} T_{\varphi_{2}}+a \bar{b} e^{i \theta_{2}} T_{\varphi_{2}} T_{\varphi_{1}}
$$

we have

$$
\left(\bar{a} b e^{i \theta_{1}}-a \bar{b} e^{i \theta_{2}}\right)\left(T_{\varphi_{1}} T_{\varphi_{2}}-T_{\varphi_{2}} T_{\varphi_{1}}\right)=0 .
$$

Hence, $T_{\varphi}$ is normal if and only if $\bar{a} b e^{i \theta_{1}}=a \bar{b} e^{i \theta_{2}}$ or $T_{\varphi_{1}} T_{\varphi_{2}}=T_{\varphi_{2}} T_{\varphi_{1}}$. By direct calculations, $T_{\varphi_{1}} T_{\varphi_{2}}=T_{\varphi_{2}} T_{\varphi_{1}}$ if and only if

$$
\begin{aligned}
& \frac{m !}{(m-n) !} \frac{(s+m-n) !}{(s+m-n-t) !} z^{s+m-n-t}+e^{i \theta_{1}} \frac{m !}{(m-n)} \frac{(t+m-n) !}{(t+m-n-s) !} z^{m-n+t-s} \\
& =\frac{s !}{(s-t) !} \frac{(s+m-t) !}{(s+m-t-n) !} z^{s+m-n-t}+e^{i \theta_{2}} \frac{s !}{(s-t) !} \frac{(s+n-t) !}{(s+n-t-m) !} z^{s+n-m-t} .
\end{aligned}
$$

If $m-n=s-t$, then

$$
m !(s+m-n) !=s !(2 m-n) !
$$

Therefore, $m=n$ and $s=t$ and so

$$
\varphi(z)=a\left(1+e^{i \theta_{1}}\right)|z|^{2 m}+b\left(1+e^{i \theta_{2}}\right)|z|^{2 s} .
$$

This completes the proof.

Example 1. Let $\varphi(z)=z^{2} \bar{z}+i z \bar{z}^{2}+2 z^{3} \bar{z}-2 i z \bar{z}^{3}$. It follows from Theorem 3 with $m=2, n=s=1$, $t=3, a=1, b=2, \theta_{1}=\frac{\pi}{2}$, and $\theta_{2}=\frac{3 \pi}{2}, T_{\varphi}$ is not normal since neither $\bar{a} b e^{i \theta_{1}} \neq a \bar{b} e^{i \theta_{2}}$ nor $m \neq n$ and $s \neq t$.

If $\varphi(z)=a\left(z^{2} \bar{z}+i z \bar{z}^{2}\right)+b\left(z^{3} \bar{z}-i z \bar{z}^{3}\right)$, then $T_{\varphi}$ is normal if and only if $\bar{a} b i=-a \bar{b} i$ and so $\bar{a} b$ is a pure imaginary number.

As some applications of Theorem 2 and 3, we get the following results. The proofs can be proved in the same way as in [6].

Corollary 2. Let $\varphi(z)=a\left(z^{m} \bar{z}^{n}+e^{i \theta} z^{n} \bar{z}^{m}\right)$ with $m>n$ and nonzero $a \in \mathbb{C}$. Then, $T_{\varphi^{2}}$ is normal if and only if $\theta=\frac{k}{2} \pi(k=\mathbb{Z})$.

Corollary 3. Let $\varphi(z)=\sum_{i=1}^{n} a_{i} \varphi_{i}(z)$ where $\varphi_{i}(z)=z^{m_{i}} \bar{z}^{n_{i}}+e^{i \theta_{i}} z^{n_{i}} \bar{z}^{m_{i}}$ with nonzero $m_{i}$ and $n_{i}$, and $\theta_{i} \in \mathbb{R}$. If $a_{i} \overline{a_{j}} e^{i \theta_{j}}=\overline{a_{i}} a_{j} e^{i \theta_{i}}$ for all $i, j$, then $T_{\varphi}$ is normal.

Funding: This research was supported by Basic Science Research Program to Research Institute for Basic Sciences (RIBS) of Jeju National University through the National Research Foundation of Korea (NRF) funded by the Ministry of Education. (2019R1A6A1A10072987)

Conflicts of Interest: The authors declare no conflict of interest.

\section{References}

1. Axler, S.; Cuckovic, Z. Commuting Toeplitz operators with harmonic symbols. Integral Equ. Oper. Theory 1991, 14, 1-12. [CrossRef]

2. Cuckovi, Z.; Curto, R.E. A new necessary condition for the hyponormality of Toeplitz operators on the Bergman space. J. Oper. Theory 2018, 79, 287-300. 
3. Gu, C.; Kang, D. Normal Toeplitz and Hankel operators with operator-valued symbols. Houst. J. Math. 2014, 40, 1155-1181.

4. Hedenmalm, H.; Korenblum, B.; Zhu, K. Theory of Bergman Spaces; Springer: New York, NY, USA, 2000.

5. Hwang, I.S. Hyponormal Toeplitz operators on the Bergman space. J. Korean Math. Soc. 2005, 42, $387-403$. [CrossRef]

6. Kim, S.; Lee, J. Normal Toeplitz operators on the Bergman spaces. Mathematics 2020, 8, 1463. [CrossRef]

7. Yousef, A. Two Problems in the Theory of Toeplitz Operators on the Bergman Space. Ph.D. Thesis, The University of Toledo, Toledo, OH, USA, 2009.

8. Brown, A.; Halmos, P.R. Algebraic properties of Toeplitz operators. J. Reine Angew. Math. 1964, $213,89-102$.

9. Zhu, K. Analysis on Fock Spaces; Springer: New York, NY, USA, 2012.

10. Ko, E.; Lee, J. Hyponormality of Toeplitz operators on the Fock spaces. Complex Var. Elliptic Equ. 2018, 64, 1-19. [CrossRef]

(C) 2020 by the author. Licensee MDPI, Basel, Switzerland. This article is an open access article distributed under the terms and conditions of the Creative Commons Attribution (CC BY) license (http://creativecommons.org/licenses/by/4.0/). 